\title{
PARTIAL LINEAR EIGENVALUE STATISTICS FOR WIGNER AND SAMPLE COVARIANCE RANDOM MATRICES
}

\author{
SEAN O'ROURKE AND ALEXANDER SOSHNIKOV
}

\begin{abstract}
Let $M_{n}$ be a $n \times n$ Wigner or sample covariance random matrix, and let $\mu_{1}\left(M_{n}\right), \mu_{2}\left(M_{n}\right), \ldots, \mu_{n}\left(M_{n}\right)$ denote the unordered eigenvalues of $M_{n}$. We study the fluctuations of the partial linear eigenvalue statistics

$$
\sum_{i=1}^{n-k} f\left(\mu_{i}\left(M_{n}\right)\right)
$$

as $n \rightarrow \infty$ for sufficiently nice test functions $f$. We consider both the case when $k$ is fixed and when $\min \{k, n-k\}$ tends to infinity with $n$.
\end{abstract}

\section{INTRODUCTION}

We consider two classic random matrix ensembles with independent entries.

\subsection{Wigner Random Matrices.}

Definition 1 (Wigner random matrix). We say $M_{n}=\frac{1}{\sqrt{n}} W_{n}=\frac{1}{\sqrt{n}}\left(w_{n i j}\right)_{1 \leq i, j \leq n}$ is a real symmetric (Hermitian) Wigner matrix of size $n$ if $M_{n}$ is a $n \times n$ real symmetric (Hermitian) matrix that satisfies the following.

(i) $\left\{w_{n i j}: 1 \leq i \leq j \leq n\right\}$ is a collection of independent random variables,

(ii) for $1 \leq i<j \leq n, w_{n i j}$ has zero mean and unit variance,

(iii) for $1 \leq i \leq n, w_{n i i}$ has zero mean and variance $\sigma^{2}$.

For a Wigner matrix $M_{n}$ of size $n$, we let $\lambda_{1}\left(M_{n}\right) \leq \lambda_{2}\left(M_{n}\right) \leq \cdots \leq \lambda_{n}\left(M_{n}\right)$ denote the ordered eigenvalues of $M_{n}$ and let $\mu_{1}\left(M_{n}\right), \mu_{2}\left(M_{n}\right), \ldots, \mu_{n}\left(M_{n}\right)$ denote the unordered eigenvalues of $M_{n}$. That is, $\mu_{i}\left(M_{n}\right)=\lambda_{\pi(i)}\left(M_{n}\right)$ for $1 \leq i \leq n$, where $\pi$ is a random permutation on $\{1,2, \ldots, n\}$, chosen uniformly, independent of $M_{n}$.

We will be interested in sequences of Wigner random matrices $\left\{M_{n}\right\}_{n \geq 1}$ that satisfy the following condition.

Definition 2 (Condition C0). For each $n \geq 1$, let $M_{n}$ be a real symmetric (Hermitian) Wigner matrix of size $n$. We say the sequence $\left\{M_{n}\right\}_{n \geq 1}$ satisfies condition C0 with exponent $p \geq 0$ if there exists $\varepsilon>0$ such that

$\lim _{n \rightarrow \infty} n^{p / 2}\left(\frac{n^{4 \varepsilon}}{n^{2}} \sum_{1 \leq i<j \leq n} \mathbb{E}\left[w_{n i j}^{4} \mathbf{1}_{\left\{\left|w_{n i j}\right|>n^{1 / 2-\varepsilon}\right\}}\right]+\frac{n^{2 \varepsilon}}{n} \sum_{i=1}^{n} \mathbb{E}\left[w_{n i i}^{2} \mathbf{1}_{\left\{\left|w_{n i i}\right|>n^{1 / 2-\varepsilon}\right\}}\right]\right)=0$,

where $\mathbf{1}_{E}$ denotes the indicator function of the event $E$.

Remark 3. For each $n \geq 1$, let $M_{n}=\frac{1}{\sqrt{n}} W_{n}=\frac{1}{\sqrt{n}}\left(w_{n i j}\right)_{1 \leq i, j \leq n}$ be a real symmetric (Hermitian) Wigner matrix of size $n$. We note that if there exists $\delta>0$ such 
that

$$
\sup _{n \geq 1,1 \leq i<j \leq n} \mathbb{E}\left|w_{n i j}\right|^{4+p+\delta}<\infty \quad \text { and } \sup _{n \geq 1,1 \leq i \leq n} \mathbb{E}\left|w_{n i i}\right|^{2+p+\delta}<\infty,
$$

then $\left\{M_{n}\right\}_{n \geq 1}$ satisfies condition $\mathbf{C 0}$ with exponent $p$. We will mostly be interested in the cases when $p=0,1$.

\subsection{Sample Covariance Random Matrices.}

Definition 4 (Sample covariance matrix). Let $A_{n}=\frac{1}{n} X_{n}^{*} X_{n}$ be an $n \times n$ matrix where $X_{n}=\left(x_{n i j}\right)_{1 \leq i, j \leq n}$. We say that $A_{n}$ is a real (complex) sample covariance matrix of size $n$ if $\left\{x_{n i j}: 1 \leq i, j \leq n\right\}$ is a collection of real (complex) independent random variables each with zero mean and unit variance. In the complex case, we also require $\mathbb{E}\left(x_{n i j}^{2}\right)=0$ for all $1 \leq i, j \leq n$.

For a sample covariance matrix $A_{n}$ of size $n$, we let $\lambda_{1}\left(A_{n}\right) \leq \lambda_{2}\left(A_{n}\right) \leq \cdots \leq$ $\lambda_{n}\left(A_{n}\right)$ denote the ordered eigenvalues of $A_{n}$ and let $\mu_{1}\left(A_{n}\right), \mu_{2}\left(A_{n}\right), \ldots, \mu_{n}\left(A_{n}\right)$ denote the unordered eigenvalues of $A_{n}$.

We will be interested in sequences of sample covariance matrices $\left\{A_{n}\right\}_{n \geq 1}$ that satisfy the following condition.

Definition 5 (Condition C1). For each $n \geq 1$, let $A_{n}=\frac{1}{n} X_{n}^{*} X_{n}$ be a real (complex) sample covariance matrix of size $n$ where $X_{n}=\left(x_{n i j}\right)_{1 \leq i, j \leq n}$. We say the sequence $\left\{A_{n}\right\}_{n \geq 1}$ satisfies condition $\mathbf{C} 1$ if the random variables $x_{n i j}, 1 \leq i, j \leq n$ have symmetric distribution for all $n \geq 1$ and there exists a constant $C_{1}$ such that

$$
\sup _{n \geq 1,1 \leq i, j \leq n} \mathbb{E}\left|x_{n i j}\right|^{p} \leq\left(C_{1} \sqrt{p}\right)^{p} \quad \text { for all } \quad p \geq 1 .
$$

1.3. Known Results. For a Hermitian $n \times n$ matrix $B$, the empirical spectral distribution (ESD) $F^{B}(x)$ of $B$ is given by

$$
F^{B}(x):=\frac{1}{n} \#\left\{1 \leq i \leq n: \lambda_{i}(B) \leq x\right\},
$$

where $\lambda_{1}(B), \lambda_{2}(B), \ldots, \lambda_{n}(B)$ denote the eigenvalues of $B$. Here $\# S$ denotes the cardinality of the set $S$.

A fundamental problem in random matrix theory is to determine the limiting distribution of the ESD as the size of the matrix tends to infinity. In the 1950s, Wigner studied the limiting ESD for a large class of random Hermitian matrices whose entries on or above the diagonal are independent 30. Under certain conditions, Wigner showed that the ESD of such a matrix converges to the semicircle law $F$ with density given by

$$
\rho(x):=\left\{\begin{array}{lr}
\frac{1}{2 \pi} \sqrt{4-x^{2}}, & -2 \leq x \leq 2 \\
0, & \text { otherwise. }
\end{array}\right.
$$

The most general form of Wigner's semicircle law assumes only the first two moments of the entries [3. Theorem 2.9].

Theorem 6 (Wigner's semicircle law). For each $n \geq 1$, let $M_{n}=\frac{1}{\sqrt{n}} W_{n}=$ $\frac{1}{\sqrt{n}}\left(w_{n i j}\right)_{1 \leq i, j \leq n}$ be a real symmetric (Hermitian) Wigner matrix of size $n$. Assume that for any $\varepsilon>0$,

$$
\lim _{n \rightarrow \infty} \frac{1}{n^{2}} \sum_{i . j=1}^{n} \mathbb{E}\left|w_{n i j}\right|^{2} \mathbf{1}_{\left\{\left|w_{n i j}\right| \geq \varepsilon \sqrt{n}\right\}}=0 .
$$


Then the ESD of $M_{n}$ converges to the semicircle law $F$ with density $\rho$ defined in (11), almost surely as $n \rightarrow \infty$. Equivalently, for any continuous, bounded function $f$,

$$
\frac{1}{n} \sum_{i=1}^{n} f\left(\lambda_{i}\left(M_{n}\right)\right) \longrightarrow \int_{-\infty}^{\infty} f(x) \rho(x) d x
$$

almost surely as $n \rightarrow \infty$.

The sample covariance case was studied by Marchenko and Pastur [23. In particular, they showed that, under certain conditions, the ESD of a sample covariance random matrix converges to $F_{\mathrm{MP}}$ with density given by

$$
\rho_{\mathrm{MP}}(x):= \begin{cases}\frac{1}{2 \pi} \sqrt{\frac{4-x}{x}}, & 0<x<4 \\ 0, & \text { otherwise. }\end{cases}
$$

The Marchenko-Pastur law is the limiting ESD for a large class of sample covariance random matrices [3, Theorem 3.10].

Theorem 7 (Marchenko-Pastur law). For each $n \geq 1$, let $A_{n}=\frac{1}{n} X_{n}^{*} X_{n}$ be a real (complex) sample covariance of size $n$, where $X_{n}=\left(x_{n i j}\right)_{1 \leq i, j \leq n}$. Assume that for any $\varepsilon>0$,

$$
\lim _{n \rightarrow \infty} \frac{1}{n^{2}} \sum_{i, j=1}^{n} \mathbb{E}\left|x_{n i j}\right|^{2} \mathbf{1}_{\left\{\left|x_{n i j}\right| \geq \varepsilon \sqrt{n}\right\}}=0 .
$$

Then the ESD of $A_{n}$ converges to the Marchenko-Pastur law $F_{\mathrm{MP}}$ almost surely as $n \rightarrow \infty$. Equivalently, for any continuous, bounded function $f$,

$$
\frac{1}{n} \sum_{i=1}^{n} f\left(\lambda_{i}\left(A_{n}\right)\right) \longrightarrow \int_{-\infty}^{\infty} f(x) \rho_{\mathrm{MP}}(x) d x
$$

almost surely as $n \rightarrow \infty$.

Theorems 6 and 7 can be viewed as random matrix theory analogues of the Law of Large Numbers from classical probability theory. Thus a Central Limit Theorem for fluctuations of linear eigenvalue statistics is a natural next step.

In 24, Shcherbina studies the fluctuations of linear eigenvalue statistics for both Wigner and sample covariance random matrices. In particular, she considers test functions $f$ from the space $\mathcal{H}_{s}$ with the norm

$$
\|f\|_{s}^{2}:=\int(1+2|l|)^{2 s}|\hat{f}(l)|^{2} d l
$$

for $s>3 / 2$, where $\hat{f}$ is the Fourier transform of $f$ defined by

$$
\hat{f}(l):=\frac{1}{\sqrt{2 \pi}} \int e^{i l x} f(x) d x .
$$

We note that if $f$ is a real-valued function with $f \in \mathcal{H}_{s}$ for $s>3 / 2$, then both $f$ and $f^{\prime}$ are continuous and bounded almost everywhere [17]. In particular, this implies that $f$ is Lipschitz.

Shcherbina obtains the following two results [24, Theorems 1 and 2].

Theorem 8 (Linear eigenvalue statistics for Wigner matrices; 24]). For each $n \geq$ 1 , let $M_{n}=\frac{1}{\sqrt{n}} W_{n}=\frac{1}{\sqrt{n}}\left(w_{n i j}\right)_{1 \leq i, j \leq n}$ be a real symmetric Wigner matrix of size 
n. Suppose $\mathbb{E}\left[w_{n i j}^{4}\right]=m_{4}$ for all $1 \leq i<j \leq n$ and all $n \geq 1$. Assume for any $\varepsilon>0$

$$
\lim _{n \rightarrow \infty}\left(\frac{1}{n} \sum_{i=1}^{n} \mathbb{E}\left|w_{n i i}\right|^{2} \mathbf{1}_{\left\{\left|w_{n i i}\right| \geq \varepsilon \sqrt{n}\right\}}+\frac{1}{n^{2}} \sum_{1 \leq i<j \leq n} \mathbb{E}\left|w_{n i j}\right|^{4} \mathbf{1}_{\left\{\left|w_{n i j}\right| \geq \varepsilon \sqrt{n}\right\}}\right)=0 .
$$

Let $f$ be a real-valued function with $\|f\|_{s}<\infty$ for some $s>3 / 2$. Then

$$
\sum_{i=1}^{n} f\left(\lambda_{i}\left(M_{n}\right)\right)-\mathbb{E} \sum_{i=1}^{n} f\left(\lambda_{i}\left(M_{n}\right)\right) \longrightarrow N\left(0, v^{2}[f]\right)
$$

in distribution as $n \rightarrow \infty$, where

$$
\begin{aligned}
v^{2}[f]:= & \frac{1}{2 \pi^{2}} \int_{-2}^{2} \int_{-2}^{2}\left(\frac{f(x)-f(y)}{x-y}\right)^{2} \frac{4-x y}{\sqrt{4-x^{2}} \sqrt{4-y^{2}}} d x d y \\
& +\frac{m_{4}-3}{2 \pi^{2}}\left(\int_{-2}^{2} f(x) \frac{2-x^{2}}{\sqrt{4-x^{2}}} d x\right)^{2}+\frac{\sigma^{2}-2}{4 \pi^{2}}\left(\int_{-2}^{2} \frac{f(x) x}{\sqrt{4-x^{2}}} d x\right)^{2} .
\end{aligned}
$$

Theorem 9 (Linear eigenvalue statistics for sample covariance matrices; 24]). For each $n \geq 1$, let $A_{n}=\frac{1}{n} X_{n}^{*} X_{n}$ be a real sample covariance matrix of size $n$, where $X_{n}=\left(x_{n i j}\right)_{1 \leq i, j \leq n}$. Suppose $\mathbb{E}\left[x_{n i j}^{4}\right]=m_{4}$ for all $1 \leq i, j \leq n$ and all $n \geq 1$. Assume there exists $\varepsilon>0$ such that

$$
\sup _{n \geq 1} \sup _{1 \leq i, j \leq n} \mathbb{E}\left|x_{n i j}\right|^{4+\varepsilon}<\infty .
$$

Let $f$ be a real-valued function with $\|f\|_{s}<\infty$ for some $s>3 / 2$. Then

$$
\sum_{i=1}^{n} f\left(\lambda_{i}\left(A_{n}\right)\right)-\mathbb{E} \sum_{i=1}^{n} f\left(\lambda_{i}\left(A_{n}\right)\right) \longrightarrow N\left(0, v_{\mathrm{SC}}^{2}[f]\right)
$$

in distribution as $n \rightarrow \infty$, where

$$
\begin{aligned}
v_{\mathrm{SC}}^{2}[f]:= & \frac{1}{2 \pi^{2}} \int_{0}^{4} \int_{0}^{4}\left(\frac{f(x)-f(y)}{x-y}\right)^{2} \frac{(4-(x-2)(y-2))}{\sqrt{4-(x-2)^{2}} \sqrt{4-(y-2)^{2}}} d x d y \\
& +\frac{m_{4}-3}{4 \pi^{2}}\left(\int_{0}^{4} \frac{x-2}{\sqrt{4-(x-2)^{2}}} d x\right)^{2} .
\end{aligned}
$$

Analogous results for other random matrix ensembles (and other classes of test functions $f$ ) have also been obtained; see for example [1, 4, 9, 10, 19, 22, 24, 25, 26, 28, and references therein.

1.4. Main Results. One can observe from Theorems 8 and 9 that the variance of the linear eigenvalue statistics does not grow to infinity in the limit $n \rightarrow \infty$ for sufficiently smooth test functions. This points to very effective cancellations between different terms of the sum and a rigidity property for the distribution of the eigenvalues.

In [18, K. Johansson considered, among other things, linear statistics

$$
S_{n}=\sum_{j=1}^{n} g\left(\theta_{j}\right)
$$


of the eigenvalues $e^{i \theta_{1}}, \ldots, e^{i \theta_{n}}$ of a random $n \times n$ unitary matrix distributed according to Haar measure on $U(n)$. He proved the CLT for such linear statistics under the optimal condition

$$
\sum_{-\infty}^{\infty}|k|\left|\hat{g}_{k}\right|^{2}<\infty
$$

where $\hat{g}_{k}$ are the Fourier coefficients of $g$, and also connected the CLT result to the Szegö asymptotic formula for Toeplitz determinants (see e.g. [14, 20, 29]). Johansson's proof relies on elaborate cancellations. In particular, it works with minor modifications for more general $\beta$ ensembles, $\beta>0$ (the Haar measure case corresponding to $\beta=2$ ).

In remark 2.1 of [18, Johansson noted that for $S_{n, 1}=\sin \theta_{1}+\ldots \sin \theta_{n-1}$, the sum of the first $n-1$ terms in (5) with $g(\theta)=\sin \theta$, the distribution of the normalized statistic

$$
\frac{S_{n, 1}-\mathbb{E} S_{n, 1}}{\sqrt{\operatorname{Var} S_{n, 1}}}
$$

does not converge to the standard normal distribution as $n \rightarrow \infty$. The argument relies on the CLT for $S_{n}$ and the following facts: $\operatorname{Var} S_{n}=1 / 2$, $\operatorname{Var} S_{n, 1} \rightarrow 1$, as $n \rightarrow \infty$, and $\left|S_{n}-S_{n, 1}\right| \leq 1$.

In this paper, we study the fluctuations of the partial linear eigenvalue statistics

$$
S_{n, k}[f]:=\sum_{i=1}^{n-k} f\left(\mu_{i}\left(M_{n}\right)\right)
$$

where $k=k(n)$ is a positive integer sequence, $f$ is a sufficiently nice test function from $\mathcal{H}_{s}, s>3 / 2$, and $\left\{M_{n}\right\}_{n \geq 1}$ is a sequence of Wigner matrices that satisfy condition C0.

Theorem 10. For each $n \geq 1$, let $M_{n}=\frac{1}{\sqrt{n}} W_{n}=\frac{1}{\sqrt{n}}\left(w_{n i j}\right)_{1 \leq i, j \leq n}$ be a real symmetric Wigner matrix of size $n$. Assume the sequence $\left\{M_{n}\right\}_{n \geq 1}$ satisfies condition C0 with exponent 0 and suppose $\mathbb{E}\left[w_{n i j}^{4}\right]=m_{4}$ for all $1 \leq i<j \leq n$ and all $n \geq 1$. Let $f$ be a real-valued, bounded Lipschitz function with $\|f\|_{s}<\infty$ for some $s>3 / 2$. Let $k$ be a fixed positive integer and let $S_{n, k}[f]$ be defined by (6). Then

$$
S_{n, k}[f]-\mathbb{E} S_{n, k}[f] \longrightarrow N\left(0, v^{2}[f]\right) *\left[-\sum_{i=1}^{k}\left[f\left(\psi_{i}\right)-\mathbb{E} f\left(\psi_{i}\right)\right]\right],
$$

in distribution as $n \rightarrow \infty$, where $\psi_{1}, \ldots, \psi_{k}$ are i.i.d. semicircle-distributed random variables and $v^{2}[f]$ is given in (3).

Theorem 11. For each $n \geq 1$, let $M_{n}=\frac{1}{\sqrt{n}} W_{n}=\frac{1}{\sqrt{n}}\left(w_{n i j}\right)_{1 \leq i, j \leq n}$ be a real symmetric Wigner matrix of size $n$. Assume the sequence $\left\{M_{n}\right\}_{n \geq 1}$ satisfies condition C0 with exponent 1 and suppose $\mathbb{E}\left[w_{n i j}^{4}\right]=m_{4}$ for all $1 \leq i<j \leq n$ and all $n \geq 1$. Let $f$ be a real-valued, bounded Lipschitz function with $\|f\|_{s}<\infty$ for some $s>3 / 2$. Let $k=k(n)$ be a positive integer sequence such that $\min \{k, n-k\} \rightarrow \infty$ as $n \rightarrow \infty$. Let $S_{n, k}[f]$ be defined by (6). Then

$$
\alpha_{n, k}\left(S_{n, k}[f]-\mathbb{E} S_{n, k}[f]\right) \longrightarrow N\left(0, d^{2}[f]\right)
$$

in distribution as $n \rightarrow \infty$, where

$$
\alpha_{n, k}:=\sqrt{\frac{n}{k(n-k)}}
$$


and $d^{2}[f]:=\operatorname{Var}[f(\psi)]$ for a semicircle-distributed random variable $\psi$.

Remark 12. One can also study the case when $n-k$ is a fixed positive integer. In the proof of Theorem 10 below, we show that if $l$ is a fixed positive integer

$$
\sum_{i=1}^{l} f\left(\mu_{i}\left(M_{n}\right)\right) \longrightarrow \sum_{i=1}^{l} f\left(\psi_{i}\right)
$$

in distribution as $n \rightarrow \infty$, where $\psi_{1}, \ldots, \psi_{l}$ are i.i.d. semicircle-distributed random variables.

It should be mentioned that a different type of partial linear eigenvalue statistic for Wigner matrices has been recently studied by Bao, Pan, and Zhou in [5]. In particular, they consider $\sum_{i=1}^{k} f\left(\lambda_{i}\left(M_{n}\right)\right)$, where $\lambda_{1}\left(M_{n}\right) \leq \lambda_{2}\left(M_{n}\right) \ldots \leq \lambda_{n}\left(M_{n}\right)$ are the ordered eigenvalues of $M_{n}$ and $k$ is proportional to $n$.

Now we turn our attention to sample covariance random matrices. Let $\left\{A_{n}\right\}_{n \geq 1}$ be a sequence of sample covariance matrices that satisfies condition C1. In this case, we consider the partial linear eigenvalue statistics

$$
T_{n, k}[f]:=\sum_{i=1}^{n-k} f\left(\mu_{i}\left(A_{n}\right)\right) .
$$

Theorem 13. For each $n \geq 1$, let $A_{n}=\frac{1}{n} X_{n}^{*} X_{n}$ be a real sample covariance matrix of size $n$ where $X_{n}=\left(x_{n i j}\right)_{1 \leq i, j \leq n}$. Assume the sequence $\left\{A_{n}\right\}_{n \geq 1}$ satisfies condition $\mathbf{C 1}$ and suppose $\mathbb{E}\left[x_{n i j}^{4}\right]=m_{4}$ for all $1 \leq i, j \leq n$ and all $n \geq 1$. Let $f$ be a real-valued, bounded Lipschitz function with $\|f\|_{s}<\infty$ for some $s>3 / 2$. Let $k$ be a fixed positive integer and let $T_{n, k}[f]$ be given by (8). Then

$$
T_{n, k}[f]-\mathbb{E} T_{n, k}[f] \longrightarrow N\left(0, v_{\mathrm{SC}}^{2}[f]\right) *\left[-\sum_{i=1}^{k}\left[f\left(\psi_{i}\right)-\mathbb{E} f\left(\psi_{i}\right)\right]\right],
$$

in distribution as $n \rightarrow \infty$, where $\psi_{1}, \ldots, \psi_{k}$ are i.i.d. Marchenko-Pastur distributed random variables and $v_{\mathrm{SC}}^{2}[f]$ is given by (4).

Theorem 14. For each $n \geq 1$, let $A_{n}=\frac{1}{n} X_{n}^{*} X_{n}$ be a real sample covariance matrix of size $n$ where $X_{n}=\left(x_{n i j}\right)_{1 \leq i, j \leq n}$. Assume the sequence $\left\{A_{n}\right\}_{n \geq 1}$ satisfies condition $\mathbf{C} 1$ and suppose $\mathbb{E}\left[x_{n i j}^{4}\right]=m_{4}$ for all $1 \leq i, j \leq n$ and all $n \geq 1$. Let $f$ be a real-valued, Lipschitz function with $\|f\|_{s}<\infty$ for some $s>3 / 2$. Let $k=k(n)$ be a positive integer sequence such that $\min \{k, n-k\} \rightarrow \infty$ as $n \rightarrow \infty$. Let $T_{n, k}[f]$ be given by (8). Then

$$
\alpha_{n, k}\left(T_{n, k}[f]-\mathbb{E} T_{n, k}[f]\right) \longrightarrow N\left(0, d_{\mathrm{SC}}^{2}[f]\right)
$$

in distribution as $n \rightarrow \infty$, where $\alpha_{n, k}$ is defined in (7) and $d_{\mathrm{SC}}^{2}[f]:=\operatorname{Var}[f(\psi)]$ for a Marchenko-Pastur distributed random variable $\psi$.

The last two theorems are valid under assumptions weaker than condition C1, since one can derive the local Marchenko-Pastur law 1 at the optimal scale under assumptions analogous to those in condition $\mathbf{C 0}$ ([1]).

\footnotetext{
${ }^{1}$ The conclusion of the Marchenko-Pastur law (Theorem [7) can be equivalently stated as $\frac{\#\left\{1 \leq i \leq n: \lambda\left(A_{n}\right) \in I\right\}}{n} \longrightarrow \int_{I} \rho_{\mathrm{MP}}(x) d x$
}

almost surely as $n \rightarrow \infty$, for any fixed interval $I$. The local Marchenko-Pastur law refers to a similar conclusion holding when the interval $I$ is allowed to change with $n$. Of particular interest 
1.5. Notation and Overview. Asymptotic notations such as $O, o, \Omega$, and so forth, are used under the assumption that $n \rightarrow \infty$. The notation $O_{C}(\cdot)$ emphasizes that the hidden constant depends on $C$.

An event $E$, which depends on $n$, is said to hold with overwhelming probability if $\mathbb{P}(E) \geq 1-O_{C}\left(n^{-C}\right)$ for every constant $C>0$. We let $E^{C}$ denote the complement of the event $E$.

The paper is organized as follows. In Section 2, we prove Theorems 10 and 11 Section 3 is devoted to Theorems 13 and 14 .

1.6. Acknowledgements. The authors would like to thank Persi Diaconis and Laszlo Erdös for useful comments. We also thank the anonymous referee for many helpful comments, corrections, and references. A.S. has been supported in part by the NSF grant DMS-1007558. S.O. has been supported by grant AFOSAR-FA9550-12-1-0083.

\section{Proof of Theorems 10 and 11}

In order to study the limiting distribution of $S_{n, k}[f]-\mathbb{E} S_{n, k}[f]$, we let $\left(\xi_{1}, \ldots, \xi_{k}\right)$ be a random sample without replacement from $\{1,2, \ldots, n\}$ independent of $M_{n}$. Then

$$
S_{n, k}[f] \stackrel{\mathrm{d}}{=} \sum_{i=1}^{n} f\left(\lambda_{i}\left(M_{n}\right)\right)-\sum_{j=1}^{k} f\left(\lambda_{\xi_{j}}\left(M_{n}\right)\right)=L_{n}[f]-\sum_{j=1}^{k} f\left(\lambda_{\xi_{j}}\left(M_{n}\right)\right),
$$

where

$$
L_{n}[f]:=\operatorname{tr}\left[f\left(M_{n}\right)\right] .
$$

We now take advantage of the following rigidity result based on [12, Theorem 2.2] and [21, Theorem 3.6]. Let $\eta_{j}=\eta_{j}^{(n)}$ be the classical location of the $j$ th eigenvalue. That is,

$$
\int_{-\infty}^{\eta_{j}} \rho(x) d x=\frac{j}{n}
$$

where $\rho$ is the density of the semicircle distribution given in (1).

Theorem 15 (Rigidity of eigenvalues). Let $M_{n}=\frac{1}{\sqrt{n}} W_{n}=\frac{1}{\sqrt{n}}\left(w_{i j}\right)_{1 \leq i, j \leq n}$ be a real symmetric Wigner matrix of size $n$. Assume there exists a constant $C_{1}$ such that

$$
\sup _{1 \leq i<j \leq n} \mathbb{E}\left[w_{i j}^{4}\right] \leq C_{1} .
$$

Then for any $0<\varepsilon<1 / 2$, there exists constants $C, c>0$ and $n_{0}$ (depending only on $C_{1}, \varepsilon$, and $\sigma$ from Definition 1) such that the event

$$
\left\{\exists j:\left|\lambda_{j}\left(M_{n}\right)-\eta_{j}\right| \geq(\log n)^{c \log \log n} n^{-2 / 3}[\min \{j, n-j+1\}]^{-1 / 3}\right\}
$$

holds with probability at most

$$
\mathbb{P}\left(\Omega_{n}^{C}\right)+C\left(\frac{n^{4 \varepsilon}}{n^{2}} \sum_{1 \leq i<j \leq n} \mathbb{E}\left[w_{i j}^{4} \mathbf{1}_{\left\{\left|w_{i j}\right|>n^{1 / 2-\varepsilon}\right\}}\right]+\frac{n^{2 \varepsilon}}{n} \sum_{i=1}^{n} \mathbb{E}\left[w_{i i}^{2} \mathbf{1}_{\left\{\left|w_{i i}\right|>n^{1 / 2-\varepsilon}\right\}}\right]\right)
$$

for any $n>n_{0}$, where the event $\Omega_{n}$ holds with overwhelming probability.

is the case when the length of the interval decreases as $n$ tends to infinity; see for instance [8] and references therein. 
The proof of Theorem [15] is based on the machinery developed in [12, 21]; we present the proof in Appendix A. For the moment, we assume Theorem 15 and complete the proof of Theorems [10 and 11.

It follows from Theorem 15 that

$$
\sum_{j=1}^{k} f\left(\lambda_{\xi_{j}}\left(M_{n}\right)\right)=\sum_{j=1}^{k} f\left(\eta_{\xi_{j}}\right)+O_{f}\left(\frac{k(\log n)^{c \log \log n}}{n^{2 / 3}}\right)
$$

with probability $1-o(1)$. Here we have used the fact that $f$ is Lipschitz.

Proof of Theorem 10, From (11), we have that

$$
L_{n}[f]-\sum_{j=1}^{k} f\left(\lambda_{\xi_{j}}\left(M_{n}\right)\right)=L_{n}[f]-\sum_{j=1}^{k} f\left(\eta_{\xi_{j}}\right)+o_{f}(1)
$$

with probability $1-o(1)$. We note that $L_{n}[f]$ and $\sum_{j=1}^{k} f\left(\eta_{\xi_{j}}\right)$ are independent.

It also follows from Theorem 15 that

$$
\mathbb{E} S_{n, k}[f]=\mathbb{E} L_{n}[f]-\mathbb{E} \sum_{j=1}^{k} f\left(\eta_{\xi_{j}}\right)+o_{f}(1)
$$

since $f$ is bounded.

By Theorem 8 , it follows that $L_{n}[f]-\mathbb{E} L_{n}[f]$ converges to a normal distribution with mean zero and variance $v^{2}[f]$. It remains to compute the limiting distribution of

$$
\sum_{j=1}^{k} f\left(\eta_{\xi_{j}}\right)-\mathbb{E} \sum_{j=1}^{k} f\left(\eta_{\xi_{j}}\right)
$$

Let $\tilde{\xi}_{1}, \ldots, \tilde{\xi}_{k}$ be i.i.d. uniform random variables on $\{1,2, \ldots, n\}$ independent of $M_{n}$. We begin by noting that

$$
\mathbb{E} \sum_{j=1}^{k} f\left(\eta_{\xi_{j}}\right)=\mathbb{E} \sum_{j=1}^{k} f\left(\eta_{\tilde{\xi}_{j}}\right) .
$$

Let $g$ be an arbitrary bounded, continuous function. Then

$$
\begin{aligned}
\mathbb{E} g\left(\sum_{j=1}^{k} f\left(\eta_{\xi_{j}}\right)\right) & =\sum_{i_{1}, \ldots i_{k} \text { distinct }} g\left(\sum_{j=1}^{k} f\left(\eta_{i_{j}}\right)\right) \frac{1}{n(n-1) \cdots(n-k+1)} \\
& =\sum_{i_{1}, \ldots i_{k} \text { distinct }} g\left(\sum_{j=1}^{k} f\left(\eta_{i_{j}}\right)\right) \frac{1}{n^{k}}+O_{g}\left(\frac{k^{2}}{n}\right) \\
& =\sum_{i_{1}, \ldots, i_{k}=1}^{n} g\left(\sum_{j=1}^{k} f\left(\eta_{i_{j}}\right)\right) \frac{1}{n^{k}}+O_{g}\left(\frac{k^{2}}{n}\right) \\
& =\mathbb{E} g\left(\sum_{j=1}^{k} f\left(\eta_{\tilde{\xi}_{j}}\right)\right)+O_{g}\left(\frac{k^{2}}{n}\right) .
\end{aligned}
$$

Therefore the limiting distribution of $\sum_{j=1}^{k} f\left(\eta_{\xi_{j}}\right)$ is the same as the limiting distribution of $\sum_{j=1}^{k} f\left(\eta_{\tilde{\xi}_{j}}\right)$. A simple computation reveals that $\eta_{\tilde{\xi}_{j}}$ converges to 
the semicircle distribution as $n \rightarrow \infty$. Since $f$ is continuous and bounded on $[-2,2]$, and $k$ is fixed, the proof of Theorem 10 is complete.

Proof of Theorem 11. Suppose $\min \{k, n-k\} \rightarrow \infty$ as $n \rightarrow \infty$. We begin by noting that

$$
S_{n, k}[f] \stackrel{\mathrm{d}}{=} \sum_{i=1}^{n-k} f\left(\lambda_{\xi_{i}}\left(M_{n}\right)\right) \stackrel{\mathrm{d}}{=} L_{n}[f]-\sum_{i=1}^{k} f\left(\lambda_{\xi_{i}}\left(M_{n}\right)\right),
$$

where $\left(\xi_{1}, \xi_{2}, \ldots, \xi_{n}\right)$ is a random sample without replacement from $\{1,2, \ldots, n\}$ independent of $M_{n}$.

Since $\alpha_{n, k}=o(1)$, it follows from Theorem 9 that

$$
\alpha_{n, k}\left(L_{n}[f]-\mathbb{E} L_{n}[f]\right) \longrightarrow 0
$$

in probability as $n \rightarrow \infty$. Therefore, it suffices to show that

$$
\alpha_{n, k} \sum_{i=1}^{k}\left[f\left(\lambda_{\xi_{i}}\left(M_{n}\right)\right)-\mathbb{E} f\left(\lambda_{\xi_{i}}\left(M_{n}\right)\right)\right] \longrightarrow N\left(0, d^{2}[f]\right)
$$

in distribution as $n \rightarrow \infty$ or

$$
\alpha_{n, k} \sum_{i=1}^{n-k}\left[f\left(\lambda_{\xi_{i}}\left(M_{n}\right)\right)-\mathbb{E} f\left(\lambda_{\xi_{i}}\left(M_{n}\right)\right)\right] \longrightarrow N\left(0, d^{2}[f]\right)
$$

in distribution as $n \rightarrow \infty$.

We will verify (12) when $k \leq n-k$ and verify (13) in the case when $k>n-k$. In the setting where the sequence $\{k(n)\}_{n \geq 1}$ alternates between the two cases, we use a sub-sequence argument since the limit in each case will be the same.

Since the argument is the same in each case, we assume $k \leq n-k$ and verify (12). In this case, $\alpha_{n, k}=O\left(k^{-1 / 2}\right)$. From condition $\mathbf{C 0}$, we find that the event (10) from Theorem 15 holds with probability $o\left(n^{-1 / 2}\right)$. Since $f$ is bounded, it follows that

$$
\alpha_{n, k} \sum_{i=1}^{k} \mathbb{E} f\left(\lambda_{\xi_{i}}\left(M_{n}\right)\right)=\alpha_{n, k} \sum_{i=1}^{k} \mathbb{E} f\left(\eta_{\xi_{i}}\right)+o_{f}(1)
$$

Therefore, by (11) and (14), it suffices to show that

$$
\alpha_{n, k} \sum_{i=1}^{k}\left[f\left(\eta_{\xi_{i}}\right)-\mathbb{E} f\left(\eta_{\xi_{i}}\right)\right] \longrightarrow N\left(0, d^{2}[f]\right)
$$

in distribution as $n \rightarrow \infty$. (15) will follow from Lemma 16 below. Indeed, since $f$ is bounded, a simple computation reveals that

$$
\operatorname{Var}\left[f\left(\eta_{\xi_{1}}\right)\right] \longrightarrow \operatorname{Var}[f(\psi)]
$$

as $n \rightarrow \infty$, where $\psi$ is a semicircle-distributed random variable.

Lemma 16. For each $n \geq 1$, let $\left(\xi_{1}^{(n)}, \ldots, \xi_{k}^{(n)}\right)$ be a discrete random sample on $[n]:=\{1,2, \ldots, n\}$, where $k=k(n)$ is a positive integer sequence such that $\min \{k, n-k\} \rightarrow \infty$ as $n \rightarrow \infty$. Define $\zeta_{i}^{(n)}:=\frac{\xi_{i}^{(n)}}{n}$ for each $n \geq 1$ and $i=$ $1,2, \ldots, k$. Let $f:[0,1] \rightarrow \mathbb{R}$ be a bounded function. Then

$$
\alpha_{n, k} \sum_{i=1}^{k}\left[f\left(\zeta_{i}^{(n)}\right)-\mathbb{E} f\left(\zeta_{i}^{(n)}\right)\right] \longrightarrow N\left(0, \beta^{2}\right)
$$


in distribution as $n \rightarrow \infty$, where

$$
\beta^{2}:=\lim _{n \rightarrow \infty} \operatorname{Var}\left[f\left(\zeta_{1}^{(n)}\right)\right]
$$

and $\alpha_{n, k}$ is defined in (7).

Lemma [16] is a direct consequence of [13, Theorem 1] (see also [15] and [16, Section 3]). For completeness we give a proof of Lemma 16 in Appendix B.

\section{Proof of Theorems 13 and 14}

In order to prove Theorems 13 and 14 we require a rigidity estimate for the eigenvalues of sample covariance random matrices. Theorem 17 below provides such an estimate and is similar to Theorem 15]

Let $\gamma_{j}=\gamma_{j}^{(n)}$ be the classical location of the $j$ th eigenvalue. That is,

$$
\int_{0}^{\gamma_{j}} \rho_{\mathrm{MP}}(x) d x=\frac{j}{n}
$$

where $\rho_{\mathrm{MP}}$ is the density of the Marchenko-Pastur law given in (2).

Theorem 17. Let $A_{n}=\frac{1}{n} X_{n}^{*} X_{n}$ be a real (complex) sample covariance matrix of size $n$ where $X_{n}=\left(x_{i j}\right)_{1 \leq i, j \leq n}$. Assume there exists a constant $C_{1}$ such that

$$
\sup _{1 \leq i, j \leq n} \mathbb{E}\left|x_{i j}\right|^{p} \leq\left(C_{1} \sqrt{p}\right)^{p} \quad \text { for all } \quad p \geq 1
$$

and suppose $x_{i j}, 1 \leq i, j \leq n$ have symmetric distribution. Then there exists constants $C, c, c_{0}, c_{1}>0$ (depending only on $C_{1}$ ) such that

$$
\mathbb{P}\left(\exists j:\left|\lambda_{j}\left(A_{n}\right)-\gamma_{j}\right| \geq C(\log n)^{c \log \log n} n^{-2 / 3}\right) \leq C \exp \left(-c_{o}(\log n)^{c_{1} \log \log n}\right)
$$

for $n$ sufficiently large.

With this rigidity estimate in hand, the proof of Theorems 13 and 14 is nearly identical to the proof of Theorems 10 and 11] we leave the details to the reader. It remains to prove Theorem 17.

We will need the following version of [7, Lemma 5.1]. It should be noted that [7, Lemma 5.1] is much more general than the version stated here. For convenience, we define

$$
\varphi_{n}:=(\log n)^{\log \log n} .
$$

Lemma 18 ([7]). Let $A_{n}=\frac{1}{n} X_{n}^{*} X_{n}$ be a real (complex) sample covariance matrix of size $n$ where $X_{n}=\left(x_{i j}\right)_{1 \leq i, j \leq n}$. Assume there exists a constant $C_{1}$ such that (16) holds. Then there exists constants $C, c, c_{0}>0$ (depending only on $C_{1}$ ) such that for any $\varphi_{n}^{c}<j<n-\varphi_{n}^{c}$,

$$
\mathbb{P}\left(\gamma_{j-\varphi_{n}^{c}} \leq \lambda_{j}\left(A_{n}\right) \leq \gamma_{j+\varphi_{n}^{c}}\right) \geq 1-\exp \left(-c_{0} \varphi_{n}\right)
$$

and

$$
\mathbb{P}\left(\frac{\left|\lambda_{j}\left(A_{n}\right)-\gamma_{j}\right|}{\gamma_{j}}>\frac{C \varphi_{n}^{c}}{j\left(1-\frac{j}{n}\right)^{1 / 3}}\right) \leq \exp \left(-c_{0} \varphi_{n}\right) .
$$

Proof of Theorem 17. By the union bound, it suffices to show that

$$
\mathbb{P}\left(\left|\lambda_{j}\left(A_{n}\right)-\gamma_{j}\right| \geq C \varphi_{n}^{c} n^{-2 / 3}\right) \leq C \exp \left(-c_{0} \varphi_{n}^{c_{1}}\right)
$$

for each $1 \leq j \leq n$. Let $0<\varepsilon<1 / 100$. We consider several cases. 
(i) If $\varepsilon n \leq j<n-\varphi_{n}^{c}$, then

$$
\left|\lambda_{j}\left(A_{n}\right)-\gamma_{j}\right| \leq \frac{C \varphi_{n}^{c} \gamma_{j}}{\varepsilon n\left(\varphi_{n}^{c} / n\right)^{1 / 3}} \leq \frac{4 C \varphi_{n}^{c}}{\varepsilon n^{2 / 3}}
$$

with probability at least $1-\exp \left(-c_{0} \varphi_{n}\right)$ by Lemma 18 .

(ii) Consider the case when $\varphi_{n}^{c}<j \leq \varepsilon n$. Using that $j \leq \varepsilon n$, we have

$$
\frac{j}{n}=\int_{0}^{\gamma_{j}} \rho_{\mathrm{MP}}(x) d x \geq \frac{\sqrt{2}}{2 \pi} \int_{0}^{\gamma_{j}} x^{-1 / 2} d x=\frac{\sqrt{2}}{\pi} \sqrt{\gamma_{j}}
$$

since $\gamma_{j} \leq 2$. Thus, we obtain the bound

$$
\gamma_{j} \leq \frac{\pi^{2} j^{2}}{2 n^{2}} \quad \text { for all } 1 \leq j \leq \varepsilon n
$$

Since $\varphi_{n}^{c}<j \leq \varepsilon n$, we combine (17) with Lemma 18 to obtain

$$
\left|\lambda_{j}\left(A_{n}\right)-\gamma_{j}\right| \leq \frac{C \pi^{2} \varphi_{n}^{c} j}{2(1-\varepsilon)^{1 / 3} n^{2}} \leq \frac{C \pi^{2} \varepsilon}{2(1-\varepsilon)^{1 / 3}} \frac{\varphi_{n}^{c}}{n}
$$

with probability at least $1-\exp \left(-c_{0} \varphi_{n}\right)$.

(iii) If $1 \leq j \leq \varphi_{n}^{c}$, then $\lambda_{j} \leq \gamma_{3 \varphi_{n}^{c}}$ with probability at least $1-\exp \left(-c_{0} \varphi_{n}\right)$ by Lemma 18. Using the bound (17) for $\gamma_{3 \varphi_{n}^{c}}$, we obtain

$$
\left|\lambda_{j}\left(A_{n}\right)-\gamma_{j}\right| \leq 2 \gamma_{3 \varphi_{n}^{c}} \leq 9 \pi^{2} \frac{\varphi_{n}^{2 c}}{n^{2}}
$$

with probability at least $1-\exp \left(-c_{0} \varphi_{n}\right)$.

(iv) Consider the final case when $n-\varphi_{n}^{c} \leq j \leq n$. First we note that for any $k \leq 3 \varphi_{n}^{c}$,

$$
\frac{3 \varphi_{n}^{c}}{n} \geq \frac{k}{n}=\int_{\gamma_{n-k}}^{4} \rho_{\mathrm{MP}}(x) d x \geq \frac{1}{2 \sqrt{2} \pi} \int_{\gamma_{n-k}}^{4} \sqrt{4-x} d x
$$

and hence

$$
\left|4-\gamma_{n-k}\right| \leq\left[\frac{9 \sqrt{2} \pi \varphi_{n}^{c}}{n}\right]^{2 / 3} \quad \text { for all } \quad k \leq 3 \varphi_{n}^{c}
$$

By Lemma 18 and the estimate above, it follows that

$$
\lambda_{j}\left(A_{n}\right) \geq \gamma_{n-3 \varphi_{n}^{c}} \geq 4-\left[\frac{9 \sqrt{2} \pi \varphi_{n}^{c}}{n}\right]^{2 / 3}
$$

with probability at least $1-\exp \left(-c_{0} \varphi_{n}\right)$. By [27, Lemma 3] and Markov's inequality, there exists constant $C^{\prime}, c^{\prime}>0$ such that

$$
\begin{aligned}
\mathbb{P}\left(\lambda_{n}\left(A_{n}\right) \geq 4+\varphi_{n}^{c} n^{-2 / 3}\right) & \leq \frac{\mathbb{E}\left[\operatorname{tr}\left(A_{n}\right)^{\left\lfloor n^{2 / 3}\right\rfloor}\right]}{\left(4+\varphi_{n}^{c} n^{-2 / 3}\right)^{\left\lfloor n^{2 / 3}\right\rfloor}} \\
& \leq C^{\prime}\left(\frac{4}{4+\varphi_{n}^{c} n^{-2 / 3}}\right)^{\left\lfloor n^{2 / 3}\right\rfloor} \\
& \leq C^{\prime} \exp \left(-c^{\prime} \varphi_{n}^{c}\right) .
\end{aligned}
$$

Combining the large deviation bound above with (19) yields

$$
\lambda_{j}=4+O\left(\frac{\varphi_{n}^{c}}{n^{2 / 3}}\right)
$$


uniformly for all $n-\varphi_{n}^{c} \leq j \leq n$ with probability $1-\exp \left(-\Omega\left(\varphi_{n}^{c}\right)\right)$. Therefore, by the triangle inequality and (18)

$$
\sup _{n-\varphi_{n}^{c} \leq j \leq n}\left|\lambda_{j}-\gamma_{j}\right|=O\left(\frac{\varphi_{n}^{c}}{n^{2 / 3}}\right)
$$

with probability $1-\exp \left(-\Omega\left(\varphi_{n}^{c}\right)\right)$.

Since the cases above cover all $1 \leq j \leq n$, the proof of Theorem[17 is complete.

\section{Appendix A. Proof of Theorem 15}

This section is devoted to the proof of Theorem 15. We will need the following version of [21, Theorem 3.6].

Theorem 19 ([21]). Let $M_{n}=\frac{1}{\sqrt{n}} W_{n}$ be a real symmetric Wigner matrix where $W_{n}=\left(w_{i j}\right)_{1 \leq i, j \leq n}$. Suppose there exists constants $C_{1}, c_{1}>0$ and $0<\varepsilon<1 / 2$ such that

$$
\sup _{1 \leq i<j \leq n} \mathbb{E}\left[w_{i j}^{4}\right] \leq C_{1} \quad \text { and } \quad \sup _{1 \leq i<j \leq n} \mathbb{P}\left(\left|w_{i j}\right|>n^{1 / 2-\varepsilon}\right) \leq e^{-n^{c_{1}}} .
$$

Then there exists constants $c>0$ and $n_{0}$ (which depend only on $C_{1}, \varepsilon$, and $\sigma$ from Definition 1) such that the event

$$
\bigcup_{j=1}^{n}\left\{\left|\lambda_{j}\left(M_{n}\right)-\eta_{j}\right| \leq(\log n)^{c \log \log n} n^{-2 / 3}[\min \{j, n-j+1\}]^{-1 / 3}\right\}
$$

holds with overwhelming probability for any $n>n_{0}$.

Proof of Theorem 15. Set $\varepsilon_{n}:=n^{1 / 2-\varepsilon}$; we remind the reader that $0<\varepsilon<1 / 2$ and hence $\varepsilon_{n} \rightarrow \infty$ as $n \rightarrow \infty$. We begin with a truncation. Let

$$
\hat{w}_{i j}:=w_{i j} \mathbf{1}_{\left\{\left|w_{i j}\right| \leq \varepsilon_{n}\right\}} \quad \text { for } \quad 1 \leq i \leq j \leq n .
$$

We define the values $\mu_{i j}:=\mathbb{E} \hat{w}_{i j}$ and $\tau_{i j}^{2}:=\mathbb{E}\left[w_{i j}^{2}\right]-\mathbb{E}\left[\hat{w}_{i j}^{2}\right]$ for $1 \leq i \leq j \leq n$. Then by (9), we have

$$
\begin{array}{r}
\sup _{1 \leq i<j \leq n}\left|\mu_{i j}\right| \leq \frac{C_{1}}{\varepsilon_{n}^{3}}, \quad \sup _{1 \leq i \leq n}\left|\mu_{i i}\right| \leq \frac{\sigma^{2}}{\varepsilon_{n}} \\
\sup _{1 \leq i<j \leq n} \tau_{i j}^{2} \leq \frac{C_{1}}{\varepsilon_{n}^{2}}, \quad \sup _{1 \leq i \leq n} \tau_{i i}^{2} \leq \sigma^{2} .
\end{array}
$$

For $1 \leq i \leq j \leq n$ define the random variable $\tilde{w}_{i j}$ as a mixture of

- $\hat{w}_{i j}$ with probability $1-\frac{\left|\mu_{i j}\right|}{\varepsilon_{n}}-\frac{\tau_{i j}^{2}}{\varepsilon_{n}^{2}}$ and

- $z_{i j}$ with probability $\frac{\left|\mu_{i j}\right|}{\varepsilon_{n}}+\frac{\tau_{i j}^{2}}{\varepsilon_{n}^{2}}$,

where $z_{i j}, 1 \leq i \leq j \leq n$ are independent Bernoulli random variables independent of $W_{n}$. Set $\tilde{w}_{j i}=\tilde{w}_{i j}$ for $1 \leq i<j \leq n$. Let $\tilde{W}_{n}=\left(\tilde{w}_{i j}\right)_{1 \leq i, j \leq n}$ and $\tilde{M}_{n}=\frac{1}{\sqrt{n}} \tilde{W}_{n}$.

We now show that there exists Bernoulli random variables $z_{i j}$ such that $\tilde{M}_{n}$ is a real symmetric Wigner matrix that satisfies

$$
\sup _{1 \leq i \leq j \leq n}\left|\tilde{w}_{i j}\right| \leq n^{1 / 2-\varepsilon / 2} \quad \text { almost surely }
$$

and

$$
\sup _{1 \leq i<j \leq n} \mathbb{E}\left[\tilde{w}_{i j}^{4}\right] \leq 513 C_{1}
$$


In particular, we will construct $z_{i j}$ to be a Bernoulli random variable, symmetric about its mean, such that its mean and second moment satisfy

$$
\begin{gathered}
0=\mu_{i j}\left(1-\frac{\left|\mu_{i j}\right|}{\varepsilon_{n}}-\frac{\tau_{i j}^{2}}{\varepsilon_{n}^{2}}\right)+\mathbb{E}\left[z_{i j}\right]\left(\frac{\left|\mu_{i j}\right|}{\varepsilon_{n}}+\frac{\tau_{i j}^{2}}{\varepsilon_{n}^{2}}\right) \\
\mathbb{E}\left[w_{i j}^{2}\right]=\left(\mathbb{E}\left[w_{i j}^{2}\right]-\tau_{i j}^{2}\right)\left(1-\frac{\left|\mu_{i j}\right|}{\varepsilon_{n}}-\frac{\tau_{i j}^{2}}{\varepsilon_{n}^{2}}\right)+\mathbb{E}\left[z_{i j}^{2}\right]\left(\frac{\left|\mu_{i j}\right|}{\varepsilon_{n}}+\frac{\tau_{i j}^{2}}{\varepsilon_{n}^{2}}\right)
\end{gathered}
$$

for $1 \leq i \leq j \leq n$. We first note that, by definition of $\tilde{w}_{i j}$, we only need to consider the case when $\frac{\left|\mu_{i j}\right|}{\varepsilon_{n}}+\frac{\tau_{i j}^{2}}{\varepsilon_{n}^{2}}>0$. Suppose $a_{i j}, b_{i j}$ are real numbers that satisfy

$$
\begin{aligned}
0 & =\mu_{i j}\left(1-\frac{\left|\mu_{i j}\right|}{\varepsilon_{n}}-\frac{\tau_{i j}^{2}}{\varepsilon_{n}^{2}}\right)+a_{i j}\left(\frac{\left|\mu_{i j}\right|}{\varepsilon_{n}}+\frac{\tau_{i j}^{2}}{\varepsilon_{n}^{2}}\right) \\
\mathbb{E}\left[w_{i j}^{2}\right] & =\left(\mathbb{E}\left[w_{i j}^{2}\right]-\tau_{i j}^{2}\right)\left(1-\frac{\left|\mu_{i j}\right|}{\varepsilon_{n}}-\frac{\tau_{i j}^{2}}{\varepsilon_{n}^{2}}\right)+b_{i j}^{2}\left(\frac{\left|\mu_{i j}\right|}{\varepsilon_{n}}+\frac{\tau_{i j}^{2}}{\varepsilon_{n}^{2}}\right) .
\end{aligned}
$$

From the first equation, we obtain

$$
\left|a_{i j}\right|\left(\frac{\left|\mu_{i j}\right|}{\varepsilon_{n}}+\frac{\tau_{i j}^{2}}{\varepsilon_{n}^{2}}\right) \leq\left|\mu_{i j}\right| .
$$

From the second equation, we have

$$
b_{i j}^{2}\left(\frac{\left|\mu_{i j}\right|}{\varepsilon_{n}}+\frac{\tau_{i j}^{2}}{\varepsilon_{n}^{2}}\right)=\left(\mathbb{E}\left[w_{i j}^{2}\right]-\tau_{i j}^{2}\right)\left(\frac{\left|\mu_{i j}\right|}{\varepsilon_{n}}+\frac{\tau_{i j}^{2}}{\varepsilon_{n}^{2}}\right)+\tau_{i j}^{2} \geq \tau_{i j}^{2} .
$$

We now note that $\tau_{i j}^{2} \geq \varepsilon_{n}\left|\mu_{i j}\right|$ by definition of $\hat{w}_{i j}$ and hence

$$
\tau_{i j}^{4}+\tau_{i j}^{2}\left|\mu_{i j}\right| \varepsilon_{n}-\left|\mu_{i j}\right|^{2} \varepsilon_{n}^{2} \geq 0 .
$$

It then follows that

$$
\tau_{i j}^{2} \geq \frac{\left|\mu_{i j}\right|^{2}}{\left(\frac{\left|\mu_{i j}\right|}{\varepsilon_{n}}+\frac{\tau_{i j}^{2}}{\varepsilon_{n}^{2}}\right)} .
$$

Combining (26), (27), and (28), we obtain

$$
b_{i j}^{2}\left(\frac{\left|\mu_{i j}\right|}{\varepsilon_{n}}+\frac{\tau_{i j}^{2}}{\varepsilon_{n}^{2}}\right) \geq \tau_{i j}^{2} \geq \frac{\mu_{i j}^{2}}{\left(\frac{\left|\mu_{i j}\right|}{\varepsilon_{n}}+\frac{\tau_{i j}^{2}}{\varepsilon_{n}^{2}}\right)} \geq a_{i j}^{2}\left(\frac{\left|\mu_{i j}\right|}{\varepsilon_{n}}+\frac{\tau_{i j}^{2}}{\varepsilon_{n}^{2}}\right)
$$

and hence $b_{i j}^{2} \geq a_{i j}^{2}$. We can now define

$$
z_{i j}:=\left\{\begin{array}{ll}
a_{i j}+\sqrt{b_{i j}^{2}-a_{i j}^{2}} & \text { with probability } 1 / 2 \\
a_{i j}-\sqrt{b_{i j}^{2}-a_{i j}^{2}} & \text { with probability } 1 / 2
\end{array} .\right.
$$

It is straightforward to verify that $z_{i j}$ has mean $a_{i j}$ and second moment $b_{i j}^{2}$.

By construction $\tilde{M}_{n}$ is a real symmetric Wigner matrix. We now verify (22) and (23). By solving equations (24) and (25) for $\mathbb{E}\left[z_{i j}\right]$ and $\mathbb{E}\left[z_{i j}^{2}\right]$ and applying the bounds (20) and (21), it follows that $\left|z_{i j}\right| \leq 4 \varepsilon_{n}$. Thus we conclude that (22) holds for $n$ sufficiently large. 
We also have for $1 \leq i<j \leq n$

$$
\begin{aligned}
\mathbb{E}\left[\tilde{w}_{i j}^{4}\right] & =\mathbb{E}\left[\hat{w}_{i j}^{4}\right]\left(1-\frac{\left|\mu_{i j}\right|}{\varepsilon_{n}}-\frac{\tau_{i j}^{2}}{\varepsilon_{n}^{2}}\right)+\mathbb{E}\left[z_{i j}^{4}\right]\left(\frac{\left|\mu_{i j}\right|}{\varepsilon_{n}}+\frac{\tau_{i j}^{2}}{\varepsilon_{n}^{2}}\right) \\
& \leq C_{1}+\left(4 \varepsilon_{n}\right)^{4} 2 \frac{C_{1}}{\varepsilon_{n}^{4}} \\
& \leq 513 C_{1}
\end{aligned}
$$

by (20) and (21). This verifies (23) and hence $\tilde{M}_{n}$ satisfies the conditions of Theorem 19 .

By Theorem [19, there exists a constant $c>0$ such that the event

$$
\Omega_{n}:=\bigcup_{j=1}^{n}\left\{\left|\lambda_{j}\left(\tilde{M}_{n}\right)-\eta_{j}\right| \leq(\log n)^{c \log \log n} n^{-2 / 3}[\min \{j, n-j+1\}]^{-1 / 3}\right\}
$$

holds with overwhelming probability. Thus we obtain

$$
\begin{gathered}
\mathbb{P}\left(\exists j:\left|\lambda_{j}\left(M_{n}\right)-\eta_{j}\right| \geq(\log n)^{c \log \log n} n^{-2 / 3}[\min \{j, n-j+1\}]^{-1 / 3}\right) \\
\leq \mathbb{P}\left(\Omega_{n}^{C}\right)+\mathbb{P}\left(M_{n} \neq \tilde{M}_{n}\right) .
\end{gathered}
$$

The proof of Theorem 15 is now complete by noting that

$$
\begin{aligned}
\mathbb{P}\left(M_{n} \neq \tilde{M}_{n}\right) & \leq \sum_{i, j=1}^{n} \mathbb{P}\left(\left|w_{i j}\right|>\varepsilon_{n}\right)+\sum_{i, j=1}^{n}\left(\frac{\left|\mu_{i j}\right|}{\varepsilon_{n}}+\frac{\tau_{i j}^{2}}{\varepsilon_{n}^{2}}\right) \\
& \leq \frac{2}{\varepsilon_{n}^{4}} \sum_{1 \leq i<j \leq n} \mathbb{E}\left[w_{i j}^{4} \mathbf{1}_{\left\{\left|w_{i j}\right|>\varepsilon_{n}\right\}}\right]+\frac{2}{\varepsilon_{n}^{2}} \sum_{i=1}^{n} \mathbb{E}\left[w_{i i}^{2} \mathbf{1}_{\left\{\left|w_{i i}\right|>\varepsilon_{n}\right\}}\right] .
\end{aligned}
$$

\section{Appendix B. Proof of Lemma 16}

In order to prove Lemma 16, we use the central limit theorem for martingale difference sequences.

Theorem 20 (Theorem 35.12 of [6]). For each $N$, suppose $Z_{N 1}, Z_{N 2}, \ldots, Z_{N r_{N}}$ is a real martingale difference sequence with respect to the increasing $\sigma$-field $\left\{\mathcal{F}_{N, j}\right\}$ having second moments. If as $N \rightarrow \infty$,

$$
\sum_{j=1}^{r_{N}} \mathbb{E}\left(Z_{N j}^{2} \mid \mathcal{F}_{N, j-1}\right) \stackrel{P}{\longrightarrow} v^{2}
$$

where $v^{2}$ is a positive constant, and for each $\epsilon>0$,

$$
\sum_{j=1}^{r_{N}} \mathbb{E}\left(Z_{N j}^{2} \mathbf{1}_{\left\{\left|Z_{N j}\right| \geq \epsilon\right\}}\right) \rightarrow 0
$$

then

$$
\sum_{j=1}^{r_{N}} Z_{N j} \stackrel{\mathcal{L}}{\longrightarrow} \mathcal{N}\left(0, v^{2}\right)
$$

We will also need a number of computations, which we collect in the following lemma. 
Lemma 21 (Computations). For each $n \geq 1$, let $\left(\xi_{1}^{(n)}, \ldots, \xi_{k}^{(n)}\right)$ be a discrete random sample on $[n]:=\{1,2, \ldots, n\}$, where $k=k(n)$ is a positive integer sequence. Define $\zeta_{i}^{(n)}:=\frac{\xi_{i}^{(n)}}{n}$ for each $n \geq 1$ and $i=1,2, \ldots, k$. Let $g:[0,1] \rightarrow \mathbb{R}$ be a bounded function. Then there exits a constant $C>0$ (depending only on the function $g$ ) such that

$$
\left|\mathbb{E}\left[\mathbb{E}_{j-1} g\left(\zeta_{j}^{(n)}\right)\right]^{2}-\left[\mathbb{E} g\left(\zeta_{1}^{(n)}\right)\right]^{2}\right| \leq \frac{C}{n-j+1}
$$

and

$$
\left|\mathbb{E}\left[\mathbb{E}_{j-1} g\left(\zeta_{j}^{(n)}\right)\right]^{4}-\left[\mathbb{E} g\left(\zeta_{1}^{(n)}\right)\right]^{4}\right| \leq \frac{C}{n-j+1}
$$

Proof. We write

$$
\begin{aligned}
\mathbb{E}\left[\mathbb{E}_{j-1} g\left(\zeta_{j}^{(n)}\right)\right]^{2}= & \mathbb{E} \sum_{s, t \notin\left\{\xi_{1}^{(n)}, \ldots, \xi_{j-1}^{(n)}\right\}} \frac{g(s / n) g(t / n)}{(n-j+1)^{2}} \\
= & \sum_{S \subset[n] ;|S|=j-1} \frac{1}{n(n-1) \cdots(n-j+2)} \sum_{s, t \notin S} \frac{g(s / n) g(t / n)}{(n-j+1)^{2}} \\
= & \sum_{s, t=1}^{n} \frac{g(s / n) g(t / n)}{(n-j+1)^{2}} \sum_{|S|=j-1 ; s, t \notin S} \frac{1}{n(n-1) \cdots(n-j+2)} \\
= & \sum_{s=1}^{n} \frac{g^{2}(s / n)}{(n-j+1)^{2}} \sum_{|S|=j-1 ; s \notin S} \frac{1}{n(n-1) \cdots(n-j+2)} \\
= & \frac{1}{n-j+1} \mathbb{E} g^{2}\left(\zeta_{1}^{(n)}\right)+\frac{n-j+2}{n-j+1} \mathbb{E}\left[g\left(\zeta_{1}^{(n)}\right) g\left(\zeta_{2}^{(n)}\right)\right] \\
= & \mathbb{E}\left[g\left(\zeta_{1}^{(n)}\right) g\left(\zeta_{2}^{(n)}\right)\right]+O_{g}\left(\frac{1}{n-j+1}\right)
\end{aligned}
$$

where the set $S$ in the sums above is an ordered set. We now note that

$$
\begin{aligned}
\mathbb{E}\left[g\left(\zeta_{1}^{(n)}\right) g\left(\zeta_{2}^{(n)}\right)\right] & =\sum_{s \neq t} \frac{g(s / n) g(t / n)}{n(n-1)} \\
& =\sum_{s \neq t} \frac{g(s / n) g(t / n)}{n^{2}}+O_{g}\left(\frac{1}{n}\right) \\
& =\sum_{s, t=1}^{n} \frac{g(s / n) g(t / n)}{n^{2}}+O_{g}\left(\frac{1}{n}\right) .
\end{aligned}
$$


Combing the estimates above yields (31). For (32), we write

$$
\begin{aligned}
\mathbb{E}\left[\mathbb{E}_{j-1} g\left(\zeta_{j}^{(n)}\right)\right]^{4} & =\mathbb{E} \sum_{t_{1}, \ldots, t_{4} \notin\left\{\xi_{1}^{(n)}, \ldots, \xi_{j-1}^{(n)}\right\}} \frac{g\left(t_{1} / n\right) \cdots g\left(t_{4} / n\right)}{(n-j+1)^{4}} \\
& =\sum_{S \subset[n] ;|S|=j-1} \frac{1}{n(n-1) \cdots(n-j+2)} \sum_{t_{1}, \ldots, t_{4} \notin S} \frac{g\left(t_{1} / n\right) \cdots g\left(t_{4} / n\right)}{(n-j+1)^{4}} \\
& =\sum_{t_{1}, \ldots, t_{4}=1}^{n} \frac{g\left(t_{1} / n\right) \cdots g\left(t_{4} / n\right)}{(n-j+1)^{4}} \sum_{|S|=j-1 ; t_{1}, \ldots, t_{4} \notin S} \frac{1}{n(n-1) \cdots(n-j+2)},
\end{aligned}
$$

where the set $S$ in the sums above is an ordered set. We now consider several cases where $t_{1}, \ldots, t_{4}$ are not distinct.

(1) When the first sum is over $t_{1}=t_{2}$ and $t_{1}, t_{3}, t_{4}$ are distinct, we obtain

$$
\begin{aligned}
\sum_{t_{1}, t_{3}, t_{4}} & \frac{g\left(t_{1} / n\right) \cdots g\left(t_{4} / n\right)}{(n-j+1)^{4}} \sum_{|S|=j-1 ; t_{1}, t_{3}, t_{4} \notin S} \frac{1}{n(n-1) \cdots(n-j+2)} \\
& =\sum_{t_{1}, t_{3}, t_{4}} \frac{g\left(t_{1} / n\right)^{2} g\left(t_{3} / n\right) g\left(t_{4} / n\right)}{(n-j+1)^{4}} \frac{(n-3) \cdots(n-j-1)}{n(n-1) \cdots(n-j+2)} \\
& =\mathbb{E}\left[g^{2}\left(\zeta_{1}^{(n)}\right) g\left(\zeta_{2}^{(n)}\right) g\left(\zeta_{3}^{(n)}\right)\right] \frac{(n-j)(n-j-1)}{(n-j+1)^{3}} \\
& =O_{g}\left(\frac{1}{n-j+1}\right) .
\end{aligned}
$$

(2) When the sum is over $t_{1}=t_{2}=t_{3} \neq t_{4}$, we have

$$
\begin{aligned}
& \sum_{t_{1} \neq t_{4}} \frac{g\left(t_{1} / n\right)^{3} g\left(t_{4} / n\right)}{(n-j+1)^{4}} \sum_{|S|=j-1 ; t_{1}, t_{4} \notin S} \frac{1}{n(n-1) \cdots(n-j+2)} \\
& \quad=\mathbb{E}\left[g\left(\zeta_{1}^{(n)}\right)^{3} g\left(\zeta_{2}^{(n)}\right)\right] \frac{(n-j+1)(n-j)}{(n-j+1)^{4}} \\
& \quad=O_{g}\left(\frac{1}{(n-j+1)^{2}}\right) .
\end{aligned}
$$

(3) When the sum is over $t_{1}=t_{2}=t_{3}=t_{4}$, we obtain

$$
\begin{aligned}
& \sum_{t_{1}=1}^{n} \frac{g\left(t_{1} / n\right)^{4}}{(n-j+1)^{4}} \sum_{|S|=j-1 ; t_{1} \notin S} \frac{1}{n(n-1) \cdots(n-j+2)} \\
& \quad=\mathbb{E}\left[g\left(\zeta_{1}^{(n)}\right)^{4}\right] \frac{1}{(n-j+1)^{3}} \\
& \quad=O_{g}\left(\frac{1}{(n-j+1)^{3}}\right) .
\end{aligned}
$$


Combining the above bounds yields

$$
\begin{aligned}
\mathbb{E} & {\left[\mathbb{E}_{j-1} g\left(\zeta_{j}^{(n)}\right)\right]^{4} } \\
= & \sum_{t_{1}, \ldots, t_{4} \text { distinct }} \frac{g\left(t_{1} / n\right) \cdots g\left(t_{4} / n\right)}{(n-j+1)^{4}} \sum_{|S|=j-1 ; t_{1}, \ldots, t_{4} \notin S} \frac{1}{n(n-1) \cdots(n-j+2)} \\
& \quad+O_{g}\left(\frac{1}{n-j+1}\right) .
\end{aligned}
$$

When $t_{1}, \ldots, t_{4}$ are distinct, we can compute the inside sum and obtain

$$
\begin{aligned}
& \mathbb{E}\left[\mathbb{E}_{j-1} g\left(\zeta_{j}^{(n)}\right)\right]^{4}=\mathbb{E}[\left.g\left(\zeta_{1}^{(n)}\right) \cdots g\left(\zeta_{4}^{(n)}\right)\right] \frac{(n-j)(n-j-1)(n-j-2)}{(n-j+1)^{3}} \\
&+O_{g}\left(\frac{1}{n-j+1}\right) \\
&=\mathbb{E}\left[g\left(\zeta_{1}^{(n)}\right) \cdots g\left(\zeta_{4}^{(n)}\right)\right]+O_{g}\left(\frac{1}{n-j+1}\right) .
\end{aligned}
$$

Lastly, we note that

$$
\begin{aligned}
\mathbb{E}\left[g\left(\zeta_{1}^{(n)}\right) \cdots g\left(\zeta_{4}^{(n)}\right)\right] & =\sum_{t_{1}, \ldots, t_{4} \text { distinct }} \frac{g\left(t_{1} / n\right) \cdots g\left(t_{4} / n\right)}{n(n-1)(n-2)(n-3)} \\
& =\sum_{t_{1}, \ldots, t_{4} \text { distinct }} \frac{g\left(t_{1} / n\right) \cdots g\left(t_{4} / n\right)}{n^{4}}+O_{g}\left(\frac{1}{n}\right) \\
& =\sum_{t_{1}, \ldots, t_{4}=1}^{n} \frac{g\left(t_{1} / n\right) \cdots g\left(t_{4} / n\right)}{n^{4}}+O_{g}\left(\frac{1}{n}\right) \\
& =\left[\mathbb{E} g\left(\zeta_{1}^{(n)}\right)\right]^{4}+O_{g}\left(\frac{1}{n}\right)
\end{aligned}
$$

and the proof of Lemma 21 is complete.

Proof of Lemma 16. We will use Theorem 20 to prove Lemma 16. We write

$$
\alpha_{n, k} \sum_{i=1}^{k}\left[f\left(\zeta_{i}^{(n)}\right)-\mathbb{E} f\left(\zeta_{i}^{(n)}\right)\right]=\sum_{j=1}^{k} Z_{n, j}
$$

where

$$
Z_{n, j}:=\alpha_{n, k} \sum_{i=1}^{k}\left[\mathbb{E}_{j} f\left(\zeta_{i}^{(n)}\right)-\mathbb{E}_{j-1} f\left(\zeta_{i}^{(n)}\right)\right]
$$

$\mathbb{E}_{j}$ denotes expectation with respect to the $\sigma$-algebra $\mathcal{F}_{n, j}$, and $\mathcal{F}_{n, j}=\sigma\left(\xi_{1}^{(n)}, \ldots, \xi_{j}^{(n)}\right)$.

By considering the cases when $i<j, i=j$, and $i>j$, we have that

$$
Z_{n, j}=\alpha_{n, k}\left[f\left(\zeta_{j}^{(n)}\right)-\mathbb{E}_{j-1} f\left(\zeta_{j}^{(n)}\right)+(k-j)\left(\mathbb{E}_{j} f\left(\zeta_{j+1}^{(n)}\right)-\mathbb{E}_{j-1} f\left(\zeta_{j}^{(n)}\right)\right)\right] .
$$


We now compute

$$
\begin{aligned}
\mathbb{E}_{j} f\left(\zeta_{j+1}^{(n)}\right) & =\sum_{t \notin\left\{\xi_{1}^{(n)}, \ldots, \xi_{j}^{(n)}\right\}} f(t / n) \frac{1}{n-j} \\
& =\left(1+\frac{1}{n-j}\right) \mathbb{E}_{j-1} f\left(\zeta_{j}^{(n)}\right)-\frac{1}{n-j} f\left(\zeta_{j}^{(n)}\right) .
\end{aligned}
$$

Thus,

$$
Z_{n, j}=\alpha_{n, k} \frac{n-k}{n-j}\left[f\left(\zeta_{j}^{(n)}\right)-\mathbb{E}_{j-1} f\left(\zeta_{j}^{(n)}\right)\right] .
$$

Since $f$ is bounded and $\alpha_{n, k}=o(1)$ it follows that $Z_{n, j}=o(1)$ uniformly for $j=1,2, \ldots, k$. So the events $\left\{\left|Z_{n, j}\right|>\epsilon\right\}$ are empty for $n$ sufficiently large. Thus (30) holds. (33)

We now verify (29) and compute the limiting variance. We note that

$$
\sum_{j=1}^{k} \mathbb{E}_{j-1}\left[Z_{n, j}^{2}\right]=\alpha_{n, k}^{2}(n-k)^{2} \sum_{j=1}^{k} \frac{1}{(n-j)^{2}}\left[E_{j-1} f^{2}\left(\zeta_{j}^{(n)}\right)-\left(E_{j-1} f\left(\zeta_{j}^{(n)}\right)\right)^{2}\right] .
$$

We will show that

$$
\alpha_{n, k}^{2}(n-k)^{2} \mathbb{E}\left|\sum_{j=1}^{k} \frac{1}{(n-j)^{2}}\left[\mathbb{E}_{j-1} f^{2}\left(\zeta_{j}^{(n)}\right)-\mathbb{E} f^{2}\left(\zeta_{1}^{(n)}\right)\right]\right| \rightarrow 0
$$

and

$$
\alpha_{n, k}^{2}(n-k)^{2} \mathbb{E}\left|\sum_{j=1}^{k} \frac{1}{(n-j)^{2}}\left[\left(\mathbb{E}_{j-1} f\left(\zeta_{j}^{(n)}\right)\right)^{2}-\left(\mathbb{E} f\left(\zeta_{1}^{(n)}\right)\right)^{2}\right]\right| \longrightarrow 0
$$

as $n \rightarrow \infty$.

For (34), it suffices to prove that

$$
\alpha_{n, k}^{2}(n-k)^{2} \sum_{j=1}^{k} \frac{1}{(n-j)^{2}} \sqrt{\mathbb{E}\left|\mathbb{E}_{j-1} f^{2}\left(\zeta_{j}^{(n)}\right)-\mathbb{E} f^{2}\left(\zeta_{1}^{(n)}\right)\right|^{2}} \longrightarrow 0 .
$$

By Lemma 21, we have that

$$
\begin{aligned}
& \alpha_{n, k}^{2}(n-k)^{2} \sum_{j=1}^{k} \frac{1}{(n-j)^{2}} \sqrt{\mathbb{E}\left|\mathbb{E}_{j-1} f^{2}\left(\zeta_{j}^{(n)}\right)-\mathbb{E} f^{2}\left(\zeta_{1}^{(n)}\right)\right|^{2}} \\
& \quad=\alpha_{n, k}^{2}(n-k)^{2} \sum_{j=1}^{k} \frac{1}{(n-j)^{2}} \sqrt{\mathbb{E}\left[\mathbb{E}_{j-1} f^{2}\left(\zeta_{j}^{(n)}\right)\right]^{2}-\left[\mathbb{E} f^{2}\left(\zeta_{1}^{(n)}\right)\right]^{2}} \\
& \leq \alpha_{n, k}^{2}(n-k)^{2} \sum_{j=1}^{k} \frac{\sqrt{C}}{(n-j)^{2.5}} \\
& \leq \frac{\sqrt{C}}{\sqrt{n-k}} \alpha_{n, k}^{2}(n-k)^{2} \sum_{j=1}^{k} \frac{1}{(n-j)^{2}} \\
& \leq \frac{\sqrt{C}}{\sqrt{n-k}} \alpha_{n, k}^{2}(n-k)^{2} \frac{k}{(n-1)(n-k)} \longrightarrow 0 .
\end{aligned}
$$


Here the last inequality comes from a comparison argument between $\sum_{j=1}^{k} \frac{1}{(n-j)^{2}}$ and an appropriate integral. This verifies (34). The proof of (35) is similar and uses (32).

Using (34) and (35), we have that

$$
\sum_{j=1}^{k} \mathbb{E}_{j-1}\left[Z_{n, j}^{2}\right]-\operatorname{Var}\left[f\left(\zeta_{1}^{(n)}\right)\right] \alpha_{n, k}^{2}(n-k)^{2} \sum_{j=1}^{k} \frac{1}{(n-j)^{2}} \longrightarrow 0
$$

in probability as $n \rightarrow \infty$. However, a comparison argument verifies that

$$
\lim _{n \rightarrow \infty} \alpha_{n, k}^{2}(n-k)^{2} \sum_{j=1}^{k} \frac{1}{(n-j)^{2}}=1
$$

and the proof of Lemma 16 is complete.

\section{REFERENCES}

[1] G. W. Anderson, O. Zeitouni, CLT for a band matrix model, Probab. Theory and Related Fields, vol. 134 (2006), 283-338.

[2] Z. D. Bai, Methodologies in Spectral Analysis of Large Dimensional Random Matrices, A Review, Statistica Sinica 9 (1999), 611-677.

[3] Z. D. Bai, J. Silverstein, Spectral analysis of large dimensional random matrices, Mathematics Monograph Series 2, Science Press, Beijing 2006.

[4] Z. D. Bai, J. Silverstein, CLT for linear spectral statistics of large-dimensional sample covariance matrix, Ann. Probab., vol. 32 (2004), 553-605.

[5] Z. Bao, G. Pan, and W. Zhou, Central limit theorem for partial linear eigenvalue statistics of Wigner matrices, available ar arXiv:1206.0508 [math.PR].

[6] P. Billingsley, Probability and Measure, 3rd edition, Wiley Series in Probability and Mathematical Statistics. Wiley, New York, 1995.

[7] P. Bourgade, H-T. Yau, J. Yin, Local Circular Law for Random Matrices, available at arXiv:1206.1449 [math.PR].

[8] C. Cacciapuoti, A. Maltsev, B. Schlein, Local Marchenko-Pastur Law at the Hard Edge of Sample Covariance Matrices, available at arXiv:1206.1730 [math-ph].

[9] P. Diaconis, M. Shahshahani, On the eigenvalues of random matrices. Studies in applied probability, J. Appl. Probab. 31A (1994), 49-62.

[10] P. Diaconis, S. N. Evans, Linear functionals of eigenvalues of random matrices, Trans. Amer. Math. Soc. vol. 353, no. 7 (2001), 2615-2633.

[11] L. Erdos, private communication.

[12] L. Erdos, H-T. Yau, J. Yin, Rigidity of Eigenvalues of Generalized Wigner Matrices, available

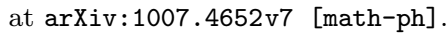

[13] P. Erdős, A. Rényi, On the central limit theorem for samples from a finite population, Magyar Tud. Akad. Mat. Kutató Int. Közl. 4 (1959), 49-61.

[14] B. L. Golinski, I. A. Ibragimov, On Szegó's limit theorem, U.S.S.R.Izv., vol. 5, no. 2 (1971), 421-446.

[15] J. Hájek, Limiting distributions in simple random sampling from a finite population, Magyar Tud. Akad. Mat. Kutató Int. Közl. 5 (1960), 361-374.

[16] J. Hájek, Collected works of Jaroslav Hájek-with commentary, Wiley Series in Probability and Statistics: Probability and Statistics. John Wiley \& Sons Ltd., Chichester, 1998. Compiled and with a preface by M. Hušková, R. Beran and V. Dupač.

[17] J. K. Hunter, B. Nachtergaele, Applied Analysis, World Scientific Publishing Co., Inc., River Edge, NJ, 2001.

[18] K. Johansson, On Szegö asymptotic formula for Toeplitz determinants and generalizations, Bull. des Sciences Mathematiques, vol. 112 (1988), 257-304.

[19] K. Johansson, On fluctuations of eigenvalues of random Hermitian matrices, Duke Math. J., vol. 91 (1998), 151-204.

[20] M. Kac, Toeplitz matrices, transation kernels and a related problem in probability theory Duke. Math. J., vol. 21 (1954) 501-509. 
[21] J. O. Lee, J. Yin, A Necessary and Sufficient Condition for Edge Universality of Wigner matrices, available at arXiv:1206.2251] [math.PR].

[22] A. Lytova, L. Pastur, Central limit theorem for linear eigenvalue statistics of random matrices with independent entries, Annals of Probability, vol. 37 (2009), 1778-1840.

[23] V. Marchenko, L. Pastur, Distribution of eigenvalues of some sets of random matrices, Math USSR-Sb. 1, (1967), 457-486.

[24] M. Shcherbina, Central Limit Theorem for linear eigenvalue statistics of the Wigner and sample covariance random matrices, available at arXiv:1101.3249 $\mathrm{v}$ [math-ph].

[25] Y. Sinai, A. Soshnikov, Central limit theorem for traces of large random symmetric matrices with independent matrix elements, Bol. Soc. Brasil. Mat. (N.S.), vol. 29 (1998), 1-24.

[26] A. Soshnikov, The central limt theorem for local linear statistics in classical compact groups and related combinatorial identities, Ann. Probab., vol. 28 (2000), 1353-1370.

[27] A. Soshnikov, A note on universality of the distribution of the largest eigenvalues in certain sample covariance matrices, J. Statist. Phys., vol 108, 1033-1056 (2002).

[28] P. Sosoe, P. Wang, Regularity conditions in the CLT for linear eigenvalue statistics of Wigner matrices, available ar arXiv:1210.5666 [math.PR].

[29] G. Szego, On certain Hermitian form associated with the Fourier series of a positive function, Comm. séminaire Math. de l'Univ. de Lund, tome supplémentaire, dédié à Marcel Riesz, (1952) 228-237.

[30] E. P. Wigner, On the distributions of the roots of certain symmetric matrices, Ann. Math. 67 (1958), 325-327.

Department of Mathematics, Yale University, New Haven, CT 06520, USA

E-mail address: sean.orourke@yale.edu

Department of Mathematics, University of California, Davis, One Shields Avenue, DAvis, CA 95616-8633

E-mail address: soshniko@math.ucdavis.edu 